\title{
Oxygen dependent pyruvate oxidase expression and production in Streptococcus sanguinis
}

\author{
Lan-yan Zheng ${ }^{1,2 *}$, Andreas Itzek ${ }^{1}$, Zhi-yun Chen $^{1}$, Jens Kreth ${ }^{1,3}$ \\ ${ }^{1}$ Department of Microbiology and Immunology, University of Oklahoma Health Sciences Center, Oklahoma 73104, \\ USA; ${ }^{2}$ China Medical University, Department of Microbiology and Parasitology, Shenyang 110001, China; \\ ${ }^{3}$ Department of Oral Biology, College of Dentistry, University of Oklahoma Health Sciences Center, Oklahoma 73104, \\ USA
}

The objective of this study was to characterize the oxygen dependent regulation of pyruvate oxidase (SpxB) gene expression and protein production in Streptococcus sanguinis (S. sanguinis). SpxB is responsible for the generation of growth-inhibiting amounts of hydrogen peroxide $\left(\mathrm{H}_{2} \mathrm{O}_{2}\right)$ able to antagonize cariogenic Streptococcus mutans (S. mutans). Furthermore, the ecological consequence of $\mathrm{H}_{2} \mathrm{O}_{2}$ production was investigated in its self-inhibiting ability towards the producing strain. Expression of $s p x B$ was determined with quantitative Real-Time RT-PCR and a fluorescent expression reporter strain. Protein abundance was investigated with FLAG epitope engineered in frame on the C-terminal end of SpxB. Self inhibition was tested with an antagonism plate assay. The expression and protein abundance decreased in cells grown under anaerobic conditions. $S$. sanguinis was resistant against its own produced $\mathrm{H}_{2} \mathrm{O}_{2}$, while cariogenic $S$. mutans was inhibited in its growth. The results suggest that $S$. sanguinis produces $\mathrm{H}_{2} \mathrm{O}_{2}$ as antimicrobial substance to inhibit susceptible niche competing species like $S$. mutans during initial biofilm formation, when oxygen availability allows for $s p x B$ expression and Spx production.

Keywords: Streptococcus sanguinis; pyruvate oxidase; oxygen dependent

International Journal of Oral Science (2011) 3: 82-89. doi: 10.4248/IJOS 11030

\section{Introduction}

Oral streptococci are important members of the oral biofilm. Their ability to initially colonize saliva-bathed tooth surfaces enables subsequent colonization by other oral bacteria [1-2]. Clinical studies suggest that successful colonization and high abundance of oral streptococci might be beneficial to oral health. For example, Streptococcus sanguinis ( $S$. sanguinis) and the cariogenic Streptococcus mutans (S. mutans) are mutually antago-

*Correspondence: Lan-yan Zheng Tel: 862423256666 ext 5315

E-mail: lyzheng@mail.cmu.edu.cn

Received 21 February 2011; Accepted 12 March 2011 nistic. Caries-free individuals have significantly higher numbers of $S$. sanguinis associated with almost nondetectable levels of $S$. mutans. This ratio changes in individuals with caries in an opposite manner [3]. Furthermore, initial colonization with abundant $S$. sanguinis delays acquisition of $S$. mutans in human subjects [4]. This information suggests an intrinsic capability of initial biofilm colonizers, comprised of up to $80 \%$ of oral streptococci [2], to promote biofilm homeostasis.

The expression of specific oral streptococcal surface proteins able to bind to salivary components is important for initial colonization and might explain the successful exclusion of pathogenic species $[1,5]$. Once a specific niche is occupied, invasion by other species might be prevented. Another important factor in the interference 
with potential pathogens is the production of antimicrobial components [6-7]. Oral streptococci, including S. sanguinis, are known to produce growth inhibiting amounts of hydrogen peroxide $\left(\mathrm{H}_{2} \mathrm{O}_{2}\right)$ [8]. Several in vitro studies demonstrate that $\mathrm{H}_{2} \mathrm{O}_{2}$ is the only agent produced by $S$. sanguinis able to inhibit the growth of $S$. mutans [6-7]. Although $S$. sanguinis also produces antimicrobial peptides [9-10], these peptides do not appear to play a role in interspecies competition with $S$. mutans.

$\mathrm{H}_{2} \mathrm{O}_{2}$ is produced by pyruvate oxidase $(\mathrm{SpxB})$ in a reaction converting pyruvate to acetyl phosphate. The reaction requires oxygen, with $\mathrm{H}_{2} \mathrm{O}_{2}$ and $\mathrm{CO}_{2}$ generated during the catalytic process [11-12]. $\mathrm{H}_{2} \mathrm{O}_{2}$ most likely diffuses out of the cell and exerts its antimicrobial effect on neighboring $\mathrm{H}_{2} \mathrm{O}_{2}$ susceptible species. Two recent studies have shown that $\operatorname{spxB}$ gene expression is controlled by at least two transcriptional regulators. Chen et al. identified several genes affecting the production of $\mathrm{H}_{2} \mathrm{O}_{2}$, including an spxR homolog [13]. SpxR was previously identified as a transcriptional activator of spxB expression in Streptococcus pneumonia (S. pneumoniae). SpxR potential binding abilities to adenosyl compounds and $\mathrm{CoA}$ containing compounds suggested an expression control of $\operatorname{spx} B$ in response to the energy and metabolic state of $S$. pneumoniae [14]. In addition, the $\operatorname{spx} B$ gene in $S$. sanguinis is under the transcriptional control of catabolite control protein A, CcpA [15]. CcpA is the main transcriptional regulator of carbon catabolite repression in Gram-positives [16].

An important environmental factor influencing the interference capability of $S$. sanguinis against $S$. mutans is oxygen. Under anaerobic conditions, $S$. sanguinis is no longer able to inhibit the growth of $S$. mutans in an in vitro antagonism assay [6]. In the present study, the effect of oxygen on $s p x B$ expression and $\mathrm{SpxB}$ abundance was further characterized. Moreover, the effect of $\mathrm{H}_{2} \mathrm{O}_{2}$ produced by $S$. sanguinis was investigated with respect to its self-inhibiting potential.

\section{Materials and Methods}

\section{Bacterial strains and media}

Bacterial strains used in this study are listed in Table 1. Strains were routinely grown aerobically as static cultures $\left(5 \% \mathrm{CO}_{2}\right)$ at $37{ }^{\circ} \mathrm{C}$ in brain heart infusion $(\mathrm{BHI}$; Difco, Sparks, MD) or on BHI agar plates. Cells were grown anaerobically in an anaerobic chamber $\left(90 \% \mathrm{~N}_{2}\right.$, $5 \% \mathrm{CO}_{2}, 5 \% \mathrm{H}_{2}$ ). Shaking cultures were grown in ambient air. Escherichia coli (E. coli) DH5a was grown at $37{ }^{\circ} \mathrm{C}$ in Luria-Bertani medium (LB, Difco) with agitation at $250 \mathrm{r} \cdot \mathrm{min}^{-1}$. When required for selection, cultures were supplemented with the following antibiotics: spectinomycin at $500 \mu \mathrm{g} \cdot \mathrm{mL}^{-1}$ for $S$. sanguinis; spectinomycin at $150 \mu \mathrm{g} \cdot \mathrm{mL}^{-1}$ and ampicillin $100 \mu \mathrm{g} \cdot \mathrm{mL}^{-1}$ for E. coli.

Table1 Strains and primers used in this study

\begin{tabular}{|c|c|c|}
\hline Strain & Relevant Characteristics & Reference \\
\hline SK36 & S. sanguinis wild type & {$[17]$} \\
\hline DL1 & S. gordonii wild type & {$[18]$} \\
\hline UA140 & S. mutans wild type & [19] \\
\hline SK36 spxB-luc & SK36::pFW5 $\Phi\left(s p x B_{p}-l u c\right)$ & {$[15]$} \\
\hline SK36 spxB-gfp & SK36::pFW5 $\Phi\left(s p x B_{p}-g f p\right)$ & This study \\
\hline SK36 spxB-FLAG & SK36::pFW5 spxB-FLAG epitope & {$[15]$} \\
\hline DH5 $\alpha$ & E. coli cloning strain & {$[20]$} \\
\hline Primer & Sequence $\left(5^{\prime} \rightarrow 3^{\prime}\right)$ & \\
\hline spxB -flag tag F & AGCGCTCGAGCGTGATTACATGAACAAACTCG & \\
\hline spxB -flag tag $\mathrm{R}$ & $\begin{array}{l}\text { CGCTAGATCTTTACTTGTCATCATCGTCTTTGTAATCTTTAATTG } \\
\text { CGCGTGATTGCA }\end{array}$ & \\
\hline Ss spxB F & AGCCGTCGACCGCAGATCCAATTGCTGT & \\
\hline Ss spxB R & AGCGGGATCCTGCTGCAGATGCAGTAAT & \\
\hline 16S rRNA F & AAGCAACGCGAAGAACCTTA & \\
\hline 16S rRNA R & GTCTCGCTAGAGTGCCCAAC & \\
\hline spxB-RT-F & AATTCGGCGGCTCAATCG & \\
\hline spxB-RT-R & AAGGATAGCAAGGAATGGAGTG & \\
\hline
\end{tabular}

www.ijos.org.cn | International Journal of Oral Science 


\section{DNA manipulations}

Standard recombinant DNA manipulations were used [21]. Restriction enzymes and DNA ligase were obtained from New England Biolabs (Beverly, MA) or Promega (Madison, WI) and used as specified by the manufacturer PCR products were cloned into the $\mathrm{pGEM}^{\circledR}-\mathrm{T}$ vector from Promega. All plasmids were extracted and purified from $E$. coli with a QIAGEN Miniprep kit (Valencia, CA). DNA extracted from agarose gels (1\%) was purified with a QIAGEN QIAquick gel extraction kit. PCR was performed with a G-Storm GS1 thermocycler (GeneTechnologies; Essex, UK) according to the manufacturer's protocol. GoTaq-DNA polymerase was obtained from Promega, and Phusion high-fidelity DNA polymerase was obtained from New England Biolabs. Primer sequences (Table 1) were designed using sequence data obtained from the Los Alamos National Laboratory Oral Pathogens Sequence Database (http://www.oralgen.lanl.gov) and synthesized by Integrated DNA Technologies (Coralville, IA).

Construction of a pyruvate oxidase carrying a $C$ terminal FLAG epitope

The FLAG epitope construction was described earlier [15]. Briefly, PCR amplification of a region about $700 \mathrm{bp}$ from the $3^{\prime}$ end of the pyruvate oxidase gene $(\operatorname{spx} B)$ was accomplished with specific primers incorporating a 6 FLAG epitope sequence (GATTACAAAGACGATGA TGACAAG) before the stop codon using primer pairs spxB-flag tag F/ spxB-flag tag R. The PCR product was inserted into the $\mathrm{pGEM}^{\mathbb{R}}-\mathrm{T}$ Easy vector (Promega) to create pGEM $^{\circledR}$-T-spxB-flag. The construct was sequenced to confirm the in-frame presence of the FLAG epitope sequence. The cloned fragment was released with Xho I/ $B g l$ II, and inserted into pFW5 [22] digested with the same enzymes. The recombinant plasmid was transformed into SK36 wild type. Transformants were selected on BHI plates containing appropriate antibiotics and were further analyzed by PCR for integration of the plasmid at the correct locus. Overnight cultures of strains carrying the Spx-FLAG construct were grown in the presence of spectinomycin.

\section{Construction of a spxB-gfp reporter strain}

A $0.7-\mathrm{kb}$ fragment containing the $\operatorname{spx} B$ promoter region was amplified by PCR from chromosomal DNA of $S$. sanguinis using primers Ss $\operatorname{spxB} \mathrm{F}$, including a Sal I linker, and Ss $s p x B \mathrm{R}$, including a $\operatorname{Bam} \mathrm{H} \mathrm{I}$ linker. The PCR product was cloned in front of the $g f p$ gene of plasmid pFW5-gfp [23] to generate plasmid pFW5spxB-GFP. The correct construct was confirmed by DNA sequencing and subsequently transformed into SK36.

\section{Western immunoblots}

Western immunoblotting was essentially performed as described earlier [15]. Briefly, after cell disruption, cytoplasmatic extracts were obtained by centrifugation and the proteins were transferred to a polyvinylidene difluoride membrane (Millipore, Bedford, MA). The membrane was blocked with a solution of 5\% skim milk dissolved in Tris-buffered saline containing $0.1 \%$ Tween 20 (TBST) for $1 \mathrm{~h}$. Subsequently, the membrane was incubated in primary antibody solution (anti-FLAG M2 antibody; Stratagene) overnight at $4{ }^{\circ} \mathrm{C}$, followed by three wash steps with TBST, and then incubated with horseradish peroxidase-conjugated secondary antibodies (Thermo Scientific) for $1 \mathrm{~h}$ at room temperature. After washing with TBST, the blots were developed using the enhanced chemiluminescence (ECL) detection system (Thermo Scientific).

\section{RNA isolation, cDNA synthesis, and real-time PCR}

RNA was isolated using QIAGEN RNeasy kit, cDNA was synthesized using Stratascript reverse transcriptase (Stratagene) according to the manufacturer's protocol. Quantitative real-time-PCR (qRT-PCR) was performed to detect specific transcripts with the comparative threshold cycle (CT) method using the Bio-Rad MyiQ Cycler (Hercules, CA). Relative changes in gene expression were calculated using the $\Delta \mathrm{CT}$ method described previously. The $16 \mathrm{~S}$ rRNA gene was used as the housekeeping reference gene. All primers used for qRT-PCR are listed in Table 1.

\section{Competition assay on solid medium}

To compare competitive growth between different oral streptococci, a protocol described previously was used with modifications [6]. Briefly, $8 \mu \mathrm{L}$ of an overnight culture of the respective species in BHI medium adjusted to an absorption at $600 \mathrm{~nm}$ of 0.8 was inoculated onto a $\mathrm{BHI}$ agar plate as the pioneer colonizer. After incubation overnight $(16 \mathrm{~h})$ at $37{ }^{\circ} \mathrm{C}$ in $5 \% \mathrm{CO}_{2}, 8 \mu \mathrm{L}$ of the competing species adjusted to an absorption at $600 \mathrm{~nm}$ of 0.8 was inoculated next to the pioneer colonizer such that the colonies almost touched each other. The plate was incubated overnight and subsequently photographed.

\section{Fluorescence microscopy}

Cells were grown in BHI on a rocking platform for maximal aeration as described earlier [6] or as static anaerobic cultures at $37{ }^{\circ} \mathrm{C}$ until late logarithmic phase $\left(A_{600 \mathrm{~nm}} \sim 0.6\right.$ to 0.7$)$. An aliquot was removed from both cultures, centrifuged at $13200 \mathrm{r} \cdot \mathrm{min}^{-1}$ in a table-top centrifuge and the liquid was replaced by fresh medium. Cells were resuspended and aerated by vigorous pipetting. 
This was performed to ensure oxygen availability to the anaerobic culture, since Gfp maturation requires oxygen presence. The cells were incubated at room temperature for $15 \mathrm{~min}$, which was shown before to be sufficient for Gfp maturation [24]. Fluorescent pictures were taken with an Olympus BX51 fluorescent microscope using appropriate fluorescent filter settings for Gfp detection and an UPlan FL N $40 \times$ or $100 \times$ oil immersion lens. Pictures were taken with Olympus DP72 digital camera and CellSens 1.3 software. Exposure times were kept constant for all images. Pictures were adjusted for brightness and contrast with photo processing software GIMP 2.6.10. (http://www.gimp.org/) applied to all parts of the image.

\section{Results}

S. sanguinis is resistant to its own $\mathrm{H}_{2} \mathrm{O}_{2}$ during competitive growth

The production of $\mathrm{H}_{2} \mathrm{O}_{2}$ by $S$. sanguinis is considered to be an antagonistic measure towards competing species during oral biofilm development. Several studies employing an in vitro antagonism assay have shown that the initial colonizer $S$. sanguinis is able to inhibit the later colonizer $S$. mutans [7, 13]. S. mutans is also able to inhibit other species by the production of bacteriocins, named mutacins [7]. S. mutans is not inhibited by its own mutacins [25], most likely due to the expression of its own resistance genes. To further confirm $\mathrm{H}_{2} \mathrm{O}_{2}$ as an antimicrobial compound produced against competing species, we tested the self-inhibiting ability against $S$. sanguinis in an antagonism plate assay. When spotted first as an initial colonizer, $S$. sanguinis was able to inhibit the growth of proximally spotted $S$. mutans. However, the growth of $S$. sanguinis and $\mathrm{H}_{2} \mathrm{O}_{2}$ producing Streptococcus gordonii (S. gordonii) was not affected. When $S$. gordonii was used as an initial colonizer, a similar result was observed, with a weak growth inhibition of $S$. sanguinis at the border of $S$. gordonii (Figure $1)$. The ability of $S$. gordonii to produce relatively more $\mathrm{H}_{2} \mathrm{O}_{2}$ compared to $S$. sanguinis might explain this observation [6]. The respective $\mathrm{SpxB}^{-}$mutants of $S$. sanguinis and $S$. gordonii did not inhibit the growth of any tested species, supporting the observation that the inhibiting substance is $\mathrm{H}_{2} \mathrm{O}_{2}$ (data not shown). In conclusion, $S$. sanguinis is able to tolerate the $\mathrm{H}_{2} \mathrm{O}_{2}$ produced both by itself and the commensal $S$. gordonii, and this observation supports the idea that $\mathrm{H}_{2} \mathrm{O}_{2}$ is produced as a competitive measure against susceptible bacteria in order to maintain biofilm homeostasis.

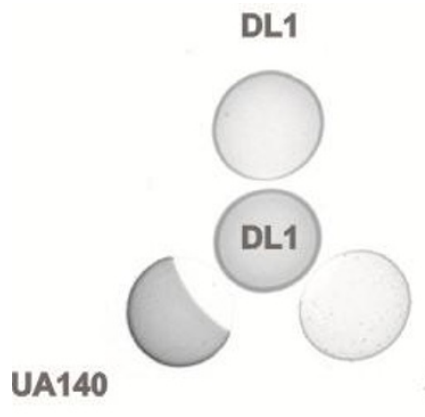

\section{SK36}

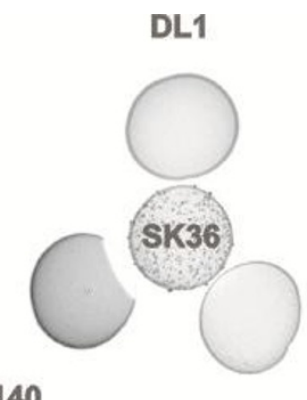

Figure 1 Antagonism assay. The strain spotted in the middle was incubated for $16 \mathrm{~h}$ before subsequent inoculation of the surrounding strains. After additional overnight incubation, growth was documented photographically.

\section{Oxygen influences $\operatorname{spxB}$ transcription}

The enzymatic conversion of pyruvate to acetyl phosphate requires oxygen [12]. As a consequence, anaerobic growth conditions diminish the ability of $S$. sanguinis to produce antagonistic amounts of $\mathrm{H}_{2} \mathrm{O}_{2}$ [6]. To test if oxygen availability influences $s p x B$ expression, the expression profile of $\operatorname{sp} x B$ was first determined using a $\operatorname{sp} x B$-luciferase reporter. As shown in Figure 2A, the relative luciferase activity of the reporter strain was similar over the measured time period, indicating that $\operatorname{spxB}$ is constitutively expressed in $S$. sanguinis under the tested growth conditions. Oxygen dependence of $\operatorname{spx} B$ expression was therefore measured with cells grown to mid-logarithmic phase as anaerobic static and aerobic shaken cultures. Using qRT-PCR, expression of sp $x B$ was increased about 25 -fold under aerobic conditions when compared to anaerobic grown cells (Figure $2 \mathrm{~B})$, suggesting that $\operatorname{sp} x B$ expression is regulated depending on oxygen availability.

\section{SpxB abundance is decreased but not absent under anaerobic conditions}

SpxB abundance was determined to test for protein production correlation with differential $\operatorname{spxB}$ expression 
under aerobic vs. anaerobic conditions. An isogenic $S$. sanguinis strain was constructed carrying a SpxB Cterminal fusion of the FLAG epitope detectable with FLAG specific antibody. The FLAG epitope did not interfere with the $\mathrm{H}_{2} \mathrm{O}_{2}$ production. Cells were grown on BHI agar plates with and without oxygen and RNA isolated as described earlier [15]. The SpxB abundance during aerobic growth was increased noticeably when compared to anaerobic grown cells (Figure 3). Interestingly, a faint band was detected for anaerobically grown cells, although no $\mathrm{H}_{2} \mathrm{O}_{2}$ was produced under these conditions [6]. A similar result was obtained with planktonic grown cells (data not shown). These results indicate that $S$. sanguinis couples $s p x B$ expression and protein production.

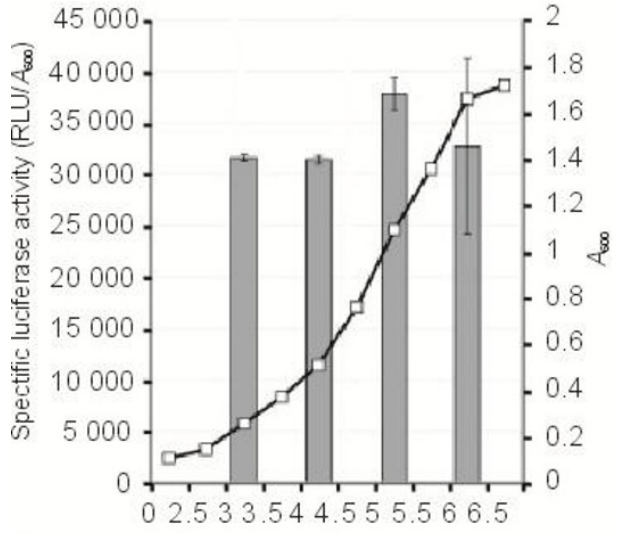

th

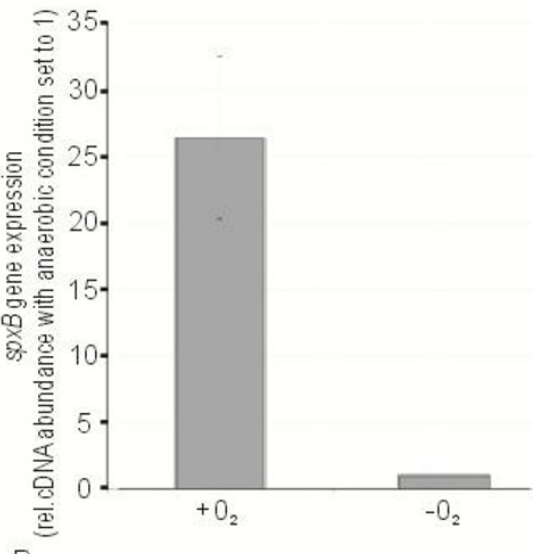

Figure 2 Growth phase and oxygen dependent regulation of $s p x B$ expression. (A) Specific luciferase activity monitored during growth of S. sanguinis. (B) qRT-PCR with cDNA prepared from aerobically and anaerobically grown cells.

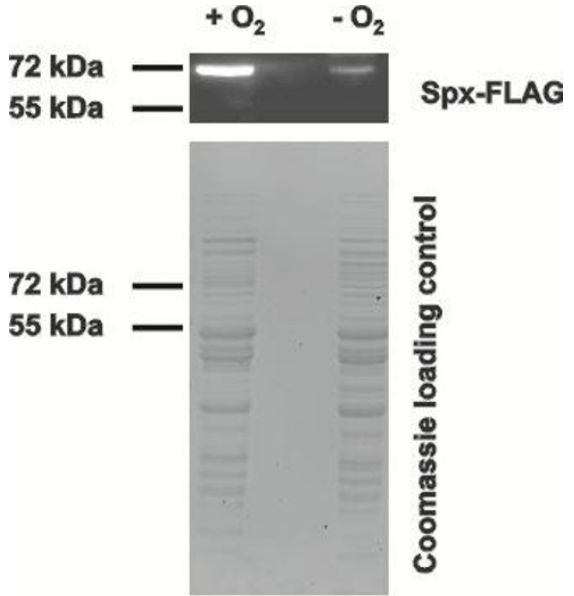

Figure 3 Effect of oxygen on SpxB abundance. Western blot analysis of SpxB protein abundance. Production of the pyruvate oxidase was detected using an antibody against the Spx-FLAG epitope. A replicate SDS gel was prepared and stained with Coomassie blue as a control. The PageRuler ${ }^{\mathrm{TM}}$ prestained protein ladder (Fermentas) was used as a size reference. Black bars depict the relative protein sizes.
Detection of spxB expression on the cellular level

Common techniques of specific gene expression analysis like qRT-PCR rely on averaging gene expression of the whole population. To determine $s p x B$ expression on the cellular level, a $s p x B-g f p$ fluorescent reporter was constructed. Planktonic cultures were grown with and without oxygen until mid-logarithmic growth phase. After aeration of the anaerobically grown culture to promote Gfp maturation, cells were analyzed with fluorescent microscopy. A weak fluorescence was detected for cells grown without oxygen, while cells grown under shaking conditions to promote aeration had a bright fluorescence (Figure 4A). The fluorescent reporter data confirm the increased expression of $s p x B$ under aerobic conditions.

After careful inspection of individual chains of aerobically grown S. sanguinis, differences in fluorescence intensity were observed in neighboring cells. As shown in Figure 4B, some neighboring cells showed bright fluorescence compared to others with a decreased fluorescence. Occasionally, single cells were observed with no fluorescence (Figure 4B, white arrow). These results suggest that at the individual cell level, differences in $s p x B$ expression exist among the investigated population. 

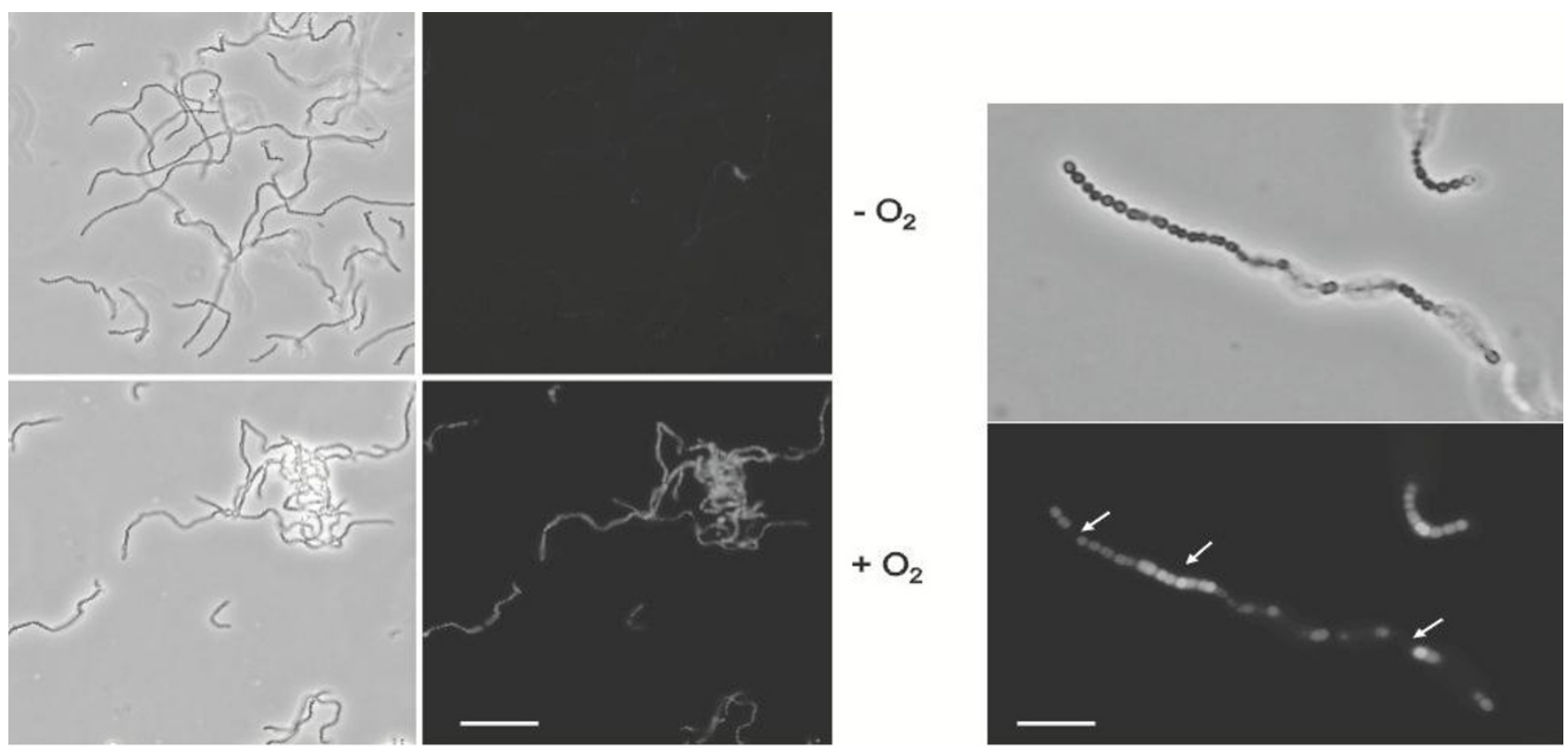

B

A Figure 4 Fluorescent microscopy of spx-gfp reporter strain grown with and without oxygen. (A) Bright field and
fluorescent image of cells grown with and without oxygen. Magnification is 400 fold. White bar $=25 \mu \mathrm{m}$. (B) Bright field and fluorescent image of cells grown with oxygen. Magnification is 1000 fold. White bar $=10 \mu \mathrm{m}$.

\section{Discussion}

In this report, the expression and protein production of the $\mathrm{H}_{2} \mathrm{O}_{2}$ generating enzyme pyruvate oxidase was further characterized in $S$. sanguinis and the consequence of $\mathrm{H}_{2} \mathrm{O}_{2}$ production on neighboring cells of its own kind was investigated. We now show that $S$. sanguinis regulates the expression of the pyruvate oxidase gene $\operatorname{spx} B$ according to the availability of oxygen. Under anaerobic conditions, $s p x B$ expression is decreased and this is also reflected in a lower abundance of SpxB. Oxygen is an important environmental factor able to influence bacterial processes in the oral biofilm $[6,26]$. It was shown previously that aerobic conditions enable S. sanguinis and $S$. gordonii to antagonize $S$. mutans [6]. Interestingly, the ability of $S$. sanguinis and $S$. gordonii to produce $\mathrm{H}_{2} \mathrm{O}_{2}$ under aerobic conditions correlates with a decreased fitness of $S$. mutans when grown in the presence of oxygen. Ahn et al. demonstrated that several phenotypic traits related to virulence properties of $S$. mutans, like biofilm formation and acid tolerance, are affected during aerobic growth. Furthermore, the ability to grow in the presence of $\mathrm{H}_{2} \mathrm{O}_{2}$ was also decreased. Although aerobically grown $S$. mutans cells were able to cope better with superoxide ions produced during treatment with paraquat, no adaptation was measured for $\mathrm{H}_{2} \mathrm{O}_{2}$ [22].
Therefore aerobically produced $\mathrm{H}_{2} \mathrm{O}_{2}$ by commensals would be the ideal antimicrobial component against susceptible species like $S$. mutans especially considering that $\mathrm{H}_{2} \mathrm{O}_{2}$ does not affect the producing cell as demonstrated in the antagonism assay (Figure 1). However, considering that oxygen tension could decrease during biofilm formation due to diffusion limitation and rapid consumption [27], $\mathrm{H}_{2} \mathrm{O}_{2}$ as an antimicrobial component would seem to be counterproductive when cells grow under anaerobic conditions. S. mutans, for example, is able to produce growth inhibiting mutacins regardless of oxygen availability [6-7]. Nonetheless, $\mathrm{H}_{2} \mathrm{O}_{2}$ production under aerobic conditions might be sufficient to function in interspecies competition. For example, an environment of high oxygen availability is most likely to occur in conditions of low biofilm abundance during initial biofilm development. As the commensals, including S. sanguinis, start to produce $\mathrm{H}_{2} \mathrm{O}_{2}$ they would naturally favor the growth of the $\mathrm{H}_{2} \mathrm{O}_{2}$ tolerant species at the expense of sensitive species, such as $S$. mutans. Another consequence of $\mathrm{H}_{2} \mathrm{O}_{2}$ production is the release of extracellular DNA (eDNA) [28], which is known to support initial biofilm formation. eDNA is an important structural component of biofilms [29] and $\mathrm{H}_{2} \mathrm{O}_{2}$ seems to be the only agent required to induce eDNA release in $S$. sanguinis and $S$. gordonii [24]. Likewise, it was shown for several species 
that eDNA is required for biofilm formation [30-32] by promoting cell-cell aggregation in S. sanguinis [28]. The $\mathrm{H}_{2} \mathrm{O}_{2}$ dependent production of eDNA might therefore promote the initial adhesion to the tooth surface and subsequent biofilm formation. Since $S$. sanguinis is an initial biofilm colonizer, its ecological fitness might be adapted to this initial stage of biofilm formation. Once the initial biofilm is established, initial colonizers might not need to produce antimicrobial components to maintain biofilm homeostasis, since the ecological niche is already occupied by up to $80 \%$ of oral streptococci [2].

The expression of $\operatorname{spx} B$ is not evenly distributed in a culture grown under optimal $\mathrm{H}_{2} \mathrm{O}_{2}$ producing conditions, although all cells should have equal access to oxygen during agitation. Non-spxB expressing cells were also detected beside cells expressing $\operatorname{spx} B$ in a relatively high abundance as visualized by Gfp fluorescence. Several recent studies have shown that bacterial populations exhibit heterogeneity in the expression of various characteristics [33-34]. For example, competence development in Bacillus subtilis occurs only in about $10 \%$ of the population [35]. This phenomenon is also referred to as bi-stability, caused by a dual pattern of gene expression in an otherwise clonal population. Another example is the occurrence of persister cells in bacterial populations. Persister cells are dormant cells in a metabolically active population and therefore highly tolerant to antibiotic treatment [33]. The sparse occurrence of $\operatorname{spxB}$ nonexpressing cells resembles metabolically inactive cells detected for $S$. sanguinis when grown under similar conditions [28]. Additional studies are required to confirm the existence of persister cells in $S$. sanguinis populations.

In conclusion, $S$. sanguinis produces $\mathrm{H}_{2} \mathrm{O}_{2}$ to antagonize cariogenic $S$. mutans, but is resistant to its own and closely related $S$. gordonii $\mathrm{H}_{2} \mathrm{O}_{2}$ in vitro. spxB expression and production of $\mathrm{SpxB}$ is dependent on oxygen availability and decreases during anaerobic growth. Ongoing studies aim to identify the transcriptional regulator responsible for the aerobic-anaerobic regulation in $S$. sanguinis to further broaden our understanding of how oral streptococci adapt to growth in the oral biofilm.

\section{Acknowledgement}

This study was supported by NIH grants 4R00DE 018400 to Jens Kreth. The authors would like to thank Dr. Joseph Ferretti (University of Oklahoma Health Sciences Center) for helpful comments.

\section{References}

1 Nobbs AH, Lamont RJ, Jenkinson HF. Streptococcus adhe- rence and colonization. Microbiol Mol Biol Rev 2009; 73: 407-450.

2 Rosan B, Lamont RJ. Dental plaque formation. Microbes Infect 2000; 2: 1599-1607.

3 Becker MR, Paster BJ, Leys EJ, et al. Molecular analysis of bacterial species associated with childhood caries. $J$ Clin Microbiol 2002; 40: 1001-1009.

4 Caufield PW, Dasanayake AP, Li Y, et al. Natural history of Streptococcus sanguinis in the oral cavity of infants: evidence for a discrete window of infectivity. Infect Immun 2000; 68: 4018-4023.

5 Gong K, Mailloux L, Herzberg MC. Salivary film expresses a complex, macromolecular binding site for Streptococcus sanguis. J Biol Chem 2000; 275: 8970-8974.

6 Kreth J, Zhang Y, Herzberg MC. Streptococcal antagonism in oral biofilms: Streptococcus sanguinis and Streptococcus gordonii interference with Streptococcus mutans. J Bacteriol 2008; 190: 4632-4640.

7 Kreth J, Merritt J, Shi W, Qi F. Competition and coexistence between Streptococcus mutans and Streptococcus sanguinis in the dental biofilm. $J$ Bacteriol 2005; 187: 7193-7203.

8 Ryan CS, Kleinberg I. Bacteria in human mouths involved in the production and utilization of hydrogen peroxide. Arch Oral Biol 1995; 40: 753-763.

9 Fujimura S, Nakamura T. Sanguicin, a bacteriocin of oral Streptococcus sanguis. Antimicrob Agents Chemother 1979; 16: 262-265.

10 Deng $\mathrm{H}$, Ding $\mathrm{Y}, \mathrm{Fu} \mathrm{MD}$, et al. Purification and characterization of sanguicin - a bacteriocin produced by Streptococcus sanguis. Sichuan Da Xие Xue Bao Yi Xue Ban 2004; 35: 555-558.

11 Carlsson J, Edlund MB. Pyruvate oxidase in Streptococcus sanguis under various growth conditions. Oral Microbiol Immunol 1987; 2: 10-14.

12 Carlsson J, Edlund MB, Lundmark SK. Characteristics of a hydrogen peroxide-forming pyruvate oxidase from Streptococcus sanguis. Oral Microbiol Immunol 1987; 2: 15-20.

13 Chen L, Ge X, Dou Y, et al. Identification of hydrogen peroxide production-related genes in Streptococcus sanguinis and their functional relationship with pyruvate oxidase. Microbiology 2011; 157: 13-20.

14 Ramos-Montanez S, Tsui HC, Wayne KJ, et al. Polymorphism and regulation of the $\operatorname{spxB}$ (pyruvate oxidase) virulence factor gene by a CBS-HotDog domain protein (SpxR) in serotype 2 Streptococcus pneumoniae. Mol Microbiol 2008; 67: 729-746.

15 Zheng L, Chen Z, Itzek A, Ashby M, Kreth J. Catabolite control protein A controls hydrogen peroxide production and cell death in Streptococcus sanguinis. J Bacteriol 2011; 193 516-526.

16 Warner JB, Lolkema JS. CcpA-dependent carbon catabolite repression in bacteria. Microbiol Mol Biol Rev 2003; 67: 
475-490.

17 Xu P, Alves JM, Kitten T, et al. Genome of the opportunistic pathogen Streptococcus sanguinis. J Bacteriol 2007; 189: 3166-3175.

18 Pakula R, Walczak W. On the nature of competence of transformable streptococci. J Gen Microbiol 1963; 31: 125133.

19 Qi F, Chen P, Caufield PW. The group I strain of Streptococcus mutans, UA140, produces both the lantibiotic mutacin I and a nonlantibiotic bacteriocin, mutacin IV . Appl Environ Microbiol 2001; 67: 15-21.

20 Hanahan D. Studies on transformation of Escherichia coli with plasmids. J Mol Biol 1983; 166: 557-580.

21 Sambrook J, Fritsch EF, Maniatis T. Molecular cloning: a laboratory manual, 2nd Edition. New York: Cold Spring Harbor Laboratory, 1989.

22 Podbielski A, Spellerberg B, Woischnik M, Pohl B, Lutticken R. Novel series of plasmid vectors for gene inactivation and expression analysis in group A streptococci (GAS). Gene 1996; 177: 137-147.

23 Kreth J, Merritt J, Shi W, Qi F. Co-ordinated bacteriocin production and competence development: a possible mechanism for taking up DNA from neighbouring species. Mol Microbiol 2005; 57: 392-404.

24 Hansen MC, Palmer RJ Jr, Udsen C, White DC, Molin S. Assessment of GFP fluorescence in cells of Streptococcus gordonii under conditions of low $\mathrm{pH}$ and low oxygen concentration. Microbiology 2001; 147: 1383-1391.

25 Tsang P, Merritt J, Nguyen T, Shi W, Qi F. Identification of genes associated with mutacin I production in Streptococcus mutans using random insertional mutagenesis. Microbiology 2005; 151: 3947-3955
26 Ahn SJ, Browngardt CM, Burne RA. Changes in biochemical and phenotypic properties of Streptococcus mutans during growth with aeration. Appl Environ Microbiol 2009; 75: $2517-2527$.

27 Socransky SS, Haffajee AD. Dental biofilms: difficult therapeutic targets. Periodontol 2000 2002; 28: 12-55.

28 Kreth J, Vu H, Zhang Y, Herzberg MC. Characterization of hydrogen peroxide-induced DNA release by Streptococcus sanguinis and Streptococcus gordonii. J Bacteriol 2009; 191: 6281-6291.

29 Flemming HC, Wingender J. The biofilm matrix. Nat Rev Microbiol 2010; 8: 623-633.

30 Dominiak DM, Nielsen JL, Nielsen PH. Extracellular DNA is abundant and important for microcolony strength in mixed microbial biofilms. Environ Microbiol 2010; 13: 710-721

31 Klein MI, DeBaz L, Agidi S, et al. Dynamics of Streptococcus mutans transcriptome in response to starch and sucrose during biofilm development. PLoS One 2010; 5: e13478.

32 Das T, Sharma PK, Busscher HJ, van der Mei HC, Krom BP Role of extracellular DNA in initial bacterial adhesion and surface aggregation. Appl Environ Microbiol 2010; 76: 3405-3408.

33 Lewis K. Persister cells. Annu Rev Microbiol 2010; 64: 357-372.

34 Veening JW, Smits WK, Kuipers OP. Bistability, epigenetics, and bet-hedging in bacteria. Annu Rev Microbiol 2008; 62: 193-210.

35 Dubnau D, Losick R. Bistability in bacteria. Mol Microbiol 2006; 61: 564-572. 